\title{
Corrections to "A Simple and Analytical Parameter-Extraction Method of a Microwave MOSFET"
}

Antonio Vilches and Ickjin Kwon

In the above paper, $Y_{\text {sub }}$ in (15) is a function of source-drain capacitance $C_{\mathrm{sd}}$, which itself is obtained from (18) after $Y_{\mathrm{sub}}$ has been evaluated. As $C_{\mathrm{sd}}$ is a function of both $R_{\mathrm{subd}}$ and $C_{\mathrm{jd}}$, which are both, in turn, functions of $Y_{\mathrm{sub}}$, the procedure in this form is not applicable. The $-j \omega C_{\mathrm{sd}}$ term is a typographical error and must be omitted from the expression. Thus, in the above paper, (15) should be

$$
\begin{aligned}
Y \mathrm{sub}= & Y_{22}-g_{\mathrm{ds}}-\omega^{2} C_{\mathrm{gd}} C_{\mathrm{dg}} R_{g}-\omega^{2} g_{m} R_{g}^{2} C_{\mathrm{gd}}\left(C_{\mathrm{gs}}+C_{\mathrm{gd}}\right) \\
& -j \omega C_{\mathrm{gd}}-j \omega g_{m} R_{g} C_{\mathrm{gd}}+j \omega^{3} C_{\mathrm{gd}} C_{\mathrm{dg}}\left(C_{\mathrm{gs}}+C_{\mathrm{gd}}\right) R_{g}^{2} \\
= & \frac{\omega^{2} C_{\mathrm{jd}}^{2} R_{\mathrm{subd}}}{1+\omega^{2} C_{\mathrm{jd}}^{2} R_{\mathrm{subd}}^{2}}+\frac{j \omega C_{\mathrm{jd}}}{1+\omega^{2} C_{\mathrm{jd}}^{2} R_{\mathrm{subd}}^{2}} .
\end{aligned}
$$

\section{REFERENCES}

[1] I. Kwon, M. Je, K. Lee, and H. Shin, "A simple and analytical parameterextraction method of a microwave MOSFET," IEEE Trans. Microwave Theory Tech., vol. 50, pp. 1503-1509, June 2002.

Manuscript received August 17, 2003; revised August 19, 2003.

A. Vilches is with the Optical and Semiconductor Devices Research Group, Department of Electrical and Electronic Engineering, Imperial College London, London SW7 2BT, U.K.

I. Kwon is with the Department of Electrical Engineering and Computer Science, Korea Advanced Institute of Science and Technology, Taejon 305-701, Korea.

Digital Object Identifier 10.1109/TMTT.2003.821223 